\title{
Revisión sobre las evidencias para la valoración de la traqueotomía durante la pandemia de COVD-19 Artículo de revisión
}

\author{
Valdés-Durán Samuel*, Santillán-Artolózaga Alberto*, Rosas-Cabral Alejandro**
}

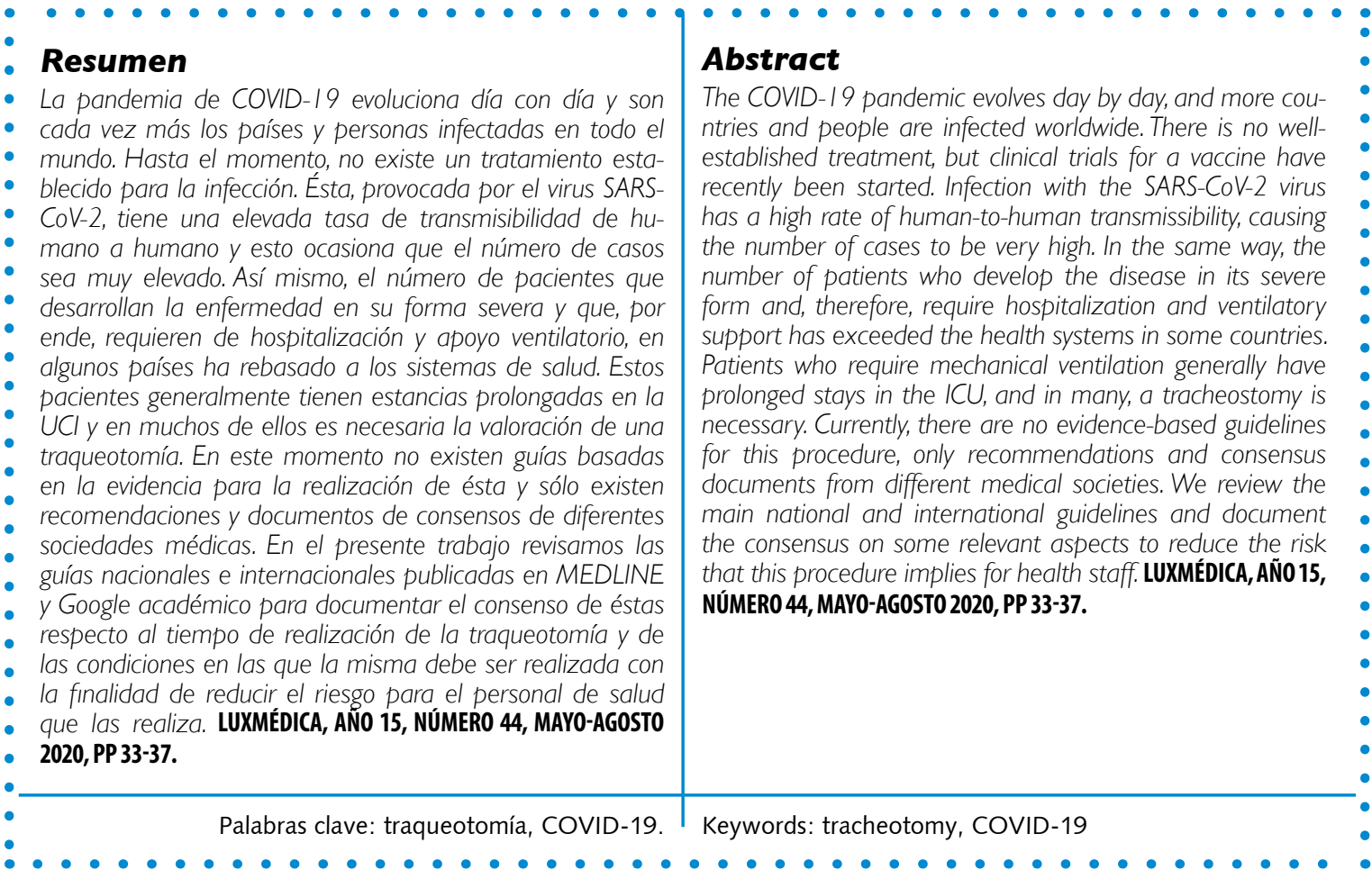

\footnotetext{
* Dr. Samuel Valdés Durán. Médico Adscrito al Servicio de Otorrinolaringología del Centenario Hospital Miguel Hidalgo, Aguascalientes, Aguascalientes, México. ORCID https://orcid.org/0000-0002-8568-8603) Correo electrónico valdesam@hotmail.com.

* Dr. Alberto Santillán Artolózaga. Médico Adscrito al Servicio de Otorrinolaringología del Centenario Hospital Miguel Hidalgo, Aguascalientes, Aguascalientes, México ORCID https://orcid.org/0000-0002-7309-5245. Correo electrónico asartolozaga@hotmail.com

${ }^{* *}$ D en C Alejandro Rosas Cabral, Departamento de Medicina, Centro de Ciencias de la Salud. Universidad Autónoma de Aguascalientes. ORCID https://orcid.org/0000-0002-6403-589X Correo electrónico drrosascabral@gmail.com

Fecha de recibido: 10 de junio 2020

Fecha de aceptación: 10 de julio 2020
}

Autor para correspondencia: Dr. Alejandro Rosas Cabral. Departamento de Medicina, Edificio 107 Planta Alta. Centro de Ciencias de la Salud. Universidad Autónoma de Aguascalientes. Avenida Universidad \# 940, Ciudad Universitaria, Código postal 20131. Aguascalientes, Aguascalientes, México. Correo electrónico drrosascabral@gmail.com 


\section{Introducción}

El brote epidémico por infección de SARS-CoV-2 en Wuhan, China, fue declarado una emergencia de salud pública internacional el 30 de enero de 2020, y la Organización Mundial de la Salud la declaró pandemia el 11 de marzo de 2020. ${ }^{1}$ En el presente trabajo revisamos las guías nacionales e internacionales publicadas en MEDLINE y Google académico para documentar el consenso de las mismas respecto al tiempo de realización de la traqueotomía y de las condiciones en las que ésta debe ser realizada con la finalidad de reducir el riesgo para el personal de salud.

\section{| | | | | | | | | | | | | | | | | | | | | | | | | | | | | | | | | | | | | | | | | | | | | | | | | | | | | | | | | | | | | | | | | | | | | | | | | | | | | | | | | | | | | | | | | | | | | | | | | | | | | | | | | | | | | | | | | | | | |}

\section{COVID 19}

El virus SARS-CoV-2 es un virus estrechamente relacionado con los coronavirus causantes del síndrome respiratorio del medio este (MERS) y el síndrome respiratorio agudo severo (SARS). ${ }^{2}$ La OMS nombró posteriormente a la enfermedad epidémica causada por el virus SARSCoV-2 como COVID-19. ${ }^{3}$ La transmisión de este virus de humano a humano ocurre principalmente entre los miembros de una familia, y en quienes tienen contacto íntimo con los pacientes o portadores asintomáticos, incluyendo al personal de la salud. El contagio a personal sanitario se reportó en China en el $3.8 \%$ del total de los casos de infección por este virus, ${ }^{4}$ aunque en Italia se reportó que el $20 \%$ de éste se infectó. ${ }^{5}$

Al momento actual, en el mundo se reportan más de 6.7 millones de casos confirmados de infección por SARS-CoV-2. En nuestro país, al 6 de junio de 2020, la Secretaría de Salud ha reportado 113,619 casos confirmados de COVID-19. ${ }^{6}$ En Aguascalientes, el Instituto de Servicios de Salud reportó para la misma fecha 1,486 casos acumulados y 58 muertes por esta causa. $^{7}$

La pandemia de COVID-19 evoluciona día a día y el número de pacientes en nuestro país va en aumento $y$, por lo tanto, los pacientes que requerirán ingreso a una Unidad de Cuidados Intensivos (UCI) también se incrementará; aunado a lo anterior, un número elevado de estos pacientes tendrán estancias hospitalarias largas y requerirán de apoyo ventilatorio por tiempo prolongado.

La experiencia inicial en la ciudad de Wuhan, sitio donde se inició la pandemia en diciembre de 2019, sugiere que aproximadamente el $12 \%$ de los pacientes con COVID-19 requirieron ingreso a la $\mathrm{UCl}$ y apoyo con ventilación mecánica. ${ }^{8}$

La tasa de mortalidad de la infección por este virus oscila entre el $0 \%$ al $14.6 \%, 9,10$ y la experiencia posterior en China reveló que entre el $9.8 \%$ y el $15.2 \%$ de los pacientes requirió ventilación mecánica. ${ }^{11-12}$ Lo anterior ha propiciado que cada vez con mayor frecuencia los intensivistas y otros especialistas implicados en el cuidado de estos pacientes tengan que decidir si al paciente se le tiene que realizar una traqueotomía.

Los beneficios y la utilidad de la traqueotomía temprana en los pacientes críticamente enfermos, antes de la pandemia de COVID-19, han sido asociados con reducción en la duración de la ventilación mecánica y en la mortalidad a corto plazo en pacientes con daño cerebral por trauma, reduciendo la estancia en $\mathrm{UCl}$ y en el hospital, además de reducir el riesgo de neumonía nosocomial, disminución del riesgo de desarrollar estenosis subglótica, disminuir el uso de sedación y mejorar la limpieza pulmonar. Sin embargo, la utilidad de ésta en pacientes con infección 
por SARS-CoV-2 se mantiene desconocida. ${ }^{13-18}$

Actualmente no existen guías basadas en la evidencia acerca de las indicaciones, tiempo óptimo o de la técnica ideal para realizar una traqueotomía, únicamente existen recomendaciones y documentos de consensos que se han limitado a la traqueotomía percutánea. ${ }^{19-21} \mathrm{La}$ traqueotomía en pacientes con COVID-19 es un procedimiento generador de aerosoles y debe ser considerada como de alto riesgo de transmisión $(\mathrm{OR}=4.15)$ para el personal de salud. ${ }^{22}$

EI SARS-CoV-2 se transmite predominantemente por gotas (5 a 10 um); sin embargo, puede formar aerosoles durante ciertas condiciones, denominadas "procedimientos generadores de aerosoles"; cuando esto sucede, las partículas virales se tornan aéreas en el núcleo de las gotas que miden menos de 5 um y pueden permanecer en el aire por más de tres horas. ${ }^{23,24}$ Cuando se presentan estas condiciones, los virus contenidos en el núcleo de la gota pueden pasar a través de las máscaras quirúrgicas y al ser inhaladas se alojan hasta el nivel alveolar. 25,26 La definición de un evento generador de aerosoles de alto riesgo no ha sido bien caracterizada a la fecha.

Los eventos generadores de aerosoles de alto riesgo son aquellos eventos que tienen el potencial de crear aerosoles con elevada carga viral y por lo tanto pueden representar un elevado riesgo para los trabajadores de la salud. Los factores que pueden aumentar el riesgo de infección incluyen la duración de la exposición, la proximidad del personal con el aerosol, la manipulación de tejidos con alta carga viral (nasofaringe/ orofaringe) y la formación de aerosoles a través del uso de dispositivos energéticos (láser, cauterio, taladros, microdebridadores, sierras o dispositivos de ultrasonido). En cuanto a la duración y proximidad de la exposición al aerosol, se ha demostrado que el mayor riesgo de transmisión fue la intubación endotraqueal $(O R=8.8)$. Tomando en cuenta que, en condiciones normales, una intubación endotraqueal dura en promedio 16 minutos desde el momento de ingresar al quirófano, cualquier exposición equivalente o mayor a este promedio debe ser considerado un factor de alto riesgo. Existe evidencia de una carga viral elevada en toda la vía aérea superior, siendo mayor en el tejido nasal que en la orofaringe. ${ }^{24,27}$

Las Guías de la Academia Americana de Otorrinolaringología establecen que, a no ser que se trate de una emergencia, a todos los pacientes que requieran algún procedimiento quirúrgico se les debe realizar previamente (48 horas antes) la prueba para COVID-1928.

Las principales guías otorrinolaringológicas actuales nacionales, ${ }^{29-31}$ al igual que las internacionales (americanas, europeas y australianas), recomiendan de manera insistente se evite la traqueotomía en pacientes COVID-19 positivos o sospechosos durante los periodos de inestabilidad respiratoria, de alta dependencia ventilatoria o con pobre pronóstico (SOFA elevado), independientemente del tiempo que tengan con intubación orotraqueal. ${ }^{19,32-38}$

La valoración para traqueotomía puede ser considerada en pacientes con estado respiratorio estable, pero no debe realizarse antes de las tres semanas de intubación, en espera de que, como factor principal, disminuya la carga viral, la cual se ha reportado que en los pacientes más graves tarda mayor tiempo en depurarse. Preferentemente, el procedimiento debe realizarse con nuevas pruebas para COVID-19 negativas (dos con diferencia de 24 horas), además de adherirse estrictamente a las normas internacionales de protección para el personal de salud, vigentes en caso de que se realice. ${ }^{29-34}$

Para poder llevar a cabo el procedimiento en cualquier paciente sin prueba previa (48 horas antes) para COVID-19 negativa o positivos sin nuevas pruebas 
de negativización (dos con diferencia de 24 horas) se requiere, además de las tres semanas de intubación, del uso por los cirujanos de trajes completos con sistema de administración de aire presurizado (PAPR) y de un quirófano con presión negativa, ya que la mascarilla N95 no es suficiente en estos casos por el mayor riesgo de generación de aerosoles con partículas mucho más pequeñas que se alcanzan a filtrar a través la máscara N95 generado por la manipulación abierta de la vía aérea. ${ }^{34,35}$

Todos los casos positivos deberán ser revisados por un equipo multidisciplinario, y los riesgos y beneficios del procedimiento para el paciente y todo el personal médico deben ser cuidadosamente evaluados. Las traqueotomías deben evitarse $o$ al menos retrasarse (tres semanas o más) debido al elevado riesgo infeccioso del procedimiento y de los cuidados subsecuentes hasta que la fase aguda de la infección haya pasado, cuando la proba- bilidad de recuperación sea alta, y cuando la progresión ventilatoria comience a ser el principal objetivo del tratamiento. Se debe evitar la traqueotomía antes de 21 días en pacientes con COVID-19 debido a la elevada virulencia; además, realizarla antes no se ha asociado con mejoría en la mortalidad ni en reducción en la estancia en la unidad de terapia intensiva, ni del tiempo en ventilación mecánica. ${ }^{37,38}$

La traqueotomía abierta en pacientes COVID-19 debe ser realizada por los cirujanos más expertos y veloces para disminuir el riesgo de contagios, y hay que señalar que no es un procedimiento exclusivo de los otorrinolaringólogos, por lo que los cirujanos de otras especialidades deberían integrarse también a los equipos de trabajo. Se debe limitar el número de participantes en la cirugía y, para proceder a realizarla, tres personas deben estar siempre presentes: dos cirujanos y un anestesiólogo altamente especializados. ${ }^{25-38}$

\section{Conclusiones}

La traqueotomía es un procedimiento generador de aerosoles de alto riesgo de transmisibilidad. Se debe realizar preferentemente en pacientes con pruebas serológicas conocidas para COVID-19. En los pacientes con COVID-19 deben evitarse o retrasarse al menos tres semanas, debido a que no se ha demostrado beneficio en la reducción de la mortalidad asociada a la ventilación mecánica en pacientes con COVID-19 y debe ser realizada por equipos especializados con el menor número de integrantes.

\section{Bibliografía}

1. Burki TK. Coronavirus in China. Lancet Resp Med. 2020; 8(3): 238. doi 10.1016/S2213-2600(20)300564.

2. Harapan $\mathrm{H}$, Itoh $\mathrm{N}$, Yufika A Winardi W, Keam $\mathrm{S}, \mathrm{Te} \mathrm{H}$ Megawati D, Hayati Z, Wagner A, Mudstair M. Coronavirus disease 2019 (COVID-19): A literature review. J Infect Public Health. 2020; 13(5): 667-673.

3. Ge H, Wang X, Yuan X, et al. The Epidemiology and clinical information about COVID-19. Eur J Clin Microbiol Infect Dis. 2020;39(6):1011-1019.

4. Guo YR, Cao QD, Hong ZS, Tan YY, Chen SD, Jin HJ, Tan KS, Wang DY, Yan Y. The origin, transmission and clinical therapies on coronavirus disease 2019 (COVID-19) outbreak - an update on the status. Mi
Med Res. 2020; 7(1):1-10. doi: 10.1186/s40779-02000240-0

5. The Lancet. COVID-19: Protecting health-care workers. 2020 Mar 21; 395(10228):922. doi: 10.1016/ S0140-6736(20)30644-9.

6. Secretaría de Salud, reporte técnico diario. Consultado 23 mayo 2020. https://www.gob.mx/cms/uploads/ attachment/file/553632/Comunicado_Tecnico_Diario COVID-19_2020.05.23.pdf

7. Instituto de Servicios de Salud del Estado de Aguascalientes. Reporte oficial diario. Consultado 23 de mayo 2020. https://www.aguascalientes.gob.mx/coronavirus/

8. Wang D, Hu B, Hu C, Zhu F, Liu X, Zhang J, Wang B, Xiang $\mathrm{H}$, Cheng $\mathrm{Z}$, Xiong $\mathrm{Y}$, et al Clinical characteristics 
of 138 hospitalized patients with 2019 novel coronavirus-infected pneumonia in Wuhan, China. JAMA 2020; 323:1061-9.

9. $X u X W, W u X X$, Jiang $X C, X u K j$, Ying $L, M a C L$, et al. Clinical findings in a group of patients infected with the 2019 novel coronavirus (SARS-CoV2) outside of Wuhan, China: retrospective case series. BMJ 2020; 368:m792.

10. Tang $N$, Deng $L$, Wang $X$, Sun Z. Abnormal coagulation parameters are associated with poor prognosis in patients with novel coronavirus pneumonia. J Thromb Haemost. 2020;18:844-847.

11. Kay JK, Chung Khoo ML, Woei Shyang L. Surgical considerations for tracheostomy during the COVID-19 pandemic. Lessons learned from the severe acute respiratory syndrome outbreak. JAMA Otolaryngol Head Neck Surg. 2020; 46(6):517-518. doi: 10.1001/jamaoto. 2020.0764

12. Wu Z, McGoogan JM: Characteristics of and important lessons from the coronavirus disease 2019 (COVID-19) outbreak in China: summary of a report of 72314 cases from the Chinese Centre for Disease Control and Prevention. JAMA 2020: 323(13):1239-1242. doi:10:1001/jama.2020.2648.

13. Raimondi N, Vial MR, Calleja J, Quintero A, Cortés A, Celis E, Pacheco C, Ugarte S, Añon JM, Hernández G et al. Evidence-based guidelines for the use of tracheostomy in critically ill patients. J Crit Care 2017; 38: 304318.

14. deFranca SA, Tavares WM, Salinet ASM, Paiva WS, Teixeira MJ. Early tracheostomy in severe traumatic brain injury patients; a meta-analysis and comparison with late tracheostomy. Crit Care Med 2020; 48(4): e325e331.

15. Michetti CP, Burlew CC, Bulger EM, Davis KA, Sapin $\mathrm{DA}$, et al. Performing tracheostomy during the Covid-19 pandemic: guidance and recommendations from the Critical care and Acute Surgery Committees of the American Association for the Surgery of Trauma. Trauma Surg Acute Care Open. 2020; 5(1):e000482.

16. Liu CC, Livingstone D, Dixon E, Dort JC. Early versus late tracheostomy: a systematic review and meta-analysis. Otolaryngol Head Neck Surg. 2015; 152(2):219227

17. Koshkareva $Y$, Gaughan JP, Soliman AM. Risk factors for adult laryngotracheal stenosis: a review of 74 cases. Ann Otol Rhinol Laryngol. 2007; 116(3):206-210.

18. Chao TN, Braslow BM, Martin ND, Challan AA, Atkins $\mathrm{JH}$, Haas AR, Rassekh $\mathrm{CH}$. Tracheotomy in ventilated patients with COVID-19. Ann Surg. 2020; 272(1):e30e32.

19. Australian New Zeland Intensive Care Society. Percutaneous dilatational tracheostomy consensus statement. http://www.anzics.com.au/Downloads/2014\% 20 The $\%$ 20ANZICS\% 20Percutaneos $\%$ 20Dilatational\% 20 Tracheostomy\% 20Consensus\% 20Statement.pdf)

20. Madsen KR, Guldager H, Rewers M, Weber SO, KobkeJacobsen K, White J. Danish guidelines 2015 for percutaneous dilatational tracheostomy in the intensive care unit. Dan Med J. 2015; 62(3):B5042

21. Raimondi N, Vial MR, Calleja J, Quintero A, Cortés A, Celis $E$, et al. Evidence-based guidelines for the use of tracheostomy in critically ill patients. Journal of Critical Care. 2017;38:304-318.

22. Engels PT, Weitzel E, Witterick IJ, Khalili S, Corsten M, Tewfik MA, Fung K, Cote D, Gupta M, Sne N, Brown TFE, James $P$, Kost KM, Sommer DD. Recommendations from the CSO-HNS Taskforce on performance of tracheotomy during the COVID-19 pandemic. Otolaryngol Head Neck Surg. 2020;49(1):23
23. van Doremalen $\mathrm{N}$, Bushmaker $\mathrm{T}$, Morris DH, Holbrook $M G$, Gamble A, Williamson BN, et al. Aerosol and surface stability of SARS-Cov-2 as compared with SARSCoV-1. NEJM. 2020; 382;1564-1567. doi:10.1056/ NEJMc2004973.

24. Howard BE. High-risk aerosol-generating procedures in COVID-19: respiratory protective equipment considerations. American Academy of Otolaryngol Head Neck Surgery Foundation 2020: doi 10.1177/0194599820927335.

25. Hang S. COVID-19: Why we should all wear masksThere is new scientific rationale. https://medium. com/@Cancerwarrior/covid-19why-we-shouldall.wear-masks-there-is-new-scientific-rationale280e08ceee71.

26. Saavedra-Mendoza AGM, Akaki-Caballero $M$, et al. Traqueotomía en pacientes con COVID-19: recomendaciones de la Sociedad Mexicana de Otorrinolaringología y Cirugía de Cabeza y Cuello. Cuando y como realizarla y cuidados posquirúrgicos. An Orl Mex 2020; 65:1-11.

27. Joseph TT, Gal JS, DeMaria S, Lin HM, Levine Al, Hyman JB. A retrospective study of success, failure, and time needed to perform awake intubation. Anesthesiology. 2016; 125(1):105-114.

28. David AP, Russell MD, El-Sayed IH, Russell MS. Tracheostomy Guidelines Developed at a Large Academic Medical Center During the COVID-19 Pandemic. Head Neck. 2020; 42(6):1291-1296. doi: 10.1002/ hed.26191.

29. Realización de la traqueotomía en pacientes con COVID-19 y el proceso de decanulación seguro (Webinar). Colegio Mexicano de Laringología y Fonocirugía / Hospital Gea González. Marzo 31,2020.

30. ¿Cómo es el manejo del paciente con COVID-19? (Webinar) Colegio Mexicano de Laringología y Fonocirugía I Instituto Nacional de Enfermedades Respiratorias. Abril 14,2020.

31. Actualizaciones útiles para el manejo de la pandemia COVID-19 (Webinar) Colegio Mexicano de Laringología y Fonocirugía. Abril 21, 2020

32. Tracheotomy in ventilated patients with COVID-19. Guidelines of the University of Pennsylvania Health System. Annals of Society of Otolaryngology-Head and Neck Surgery. Marzo 22, 2020.

33. Michigan Medicine Tracheostomy Guidelines in COVID-19 Era. Michigan University School of Medicine. Abril 1, 2020

34. Guidelines from the Stanford Group. Stanford University School of Medicine. Marzo 20, 2020.

35. Safety Recommendations for evaluation and surgery of the head and neck during the COVID-19 pandemic. JAMA Otolaryngol-Head and Neck Surgery. Marzo 31, 2020.

36. Tracheostomy guidance during the COVID-19 pandemic. British Association of Otorhinolaryngology-Head and Neck Surgery. Marzo 19, 2020.

37. Bernal-Sprekelsen $M$, Avilés-Jurado FX, Álvarez Escudero J, Alvarez-Santuyano CA, de Haro López C, Díaz de Cerio Canduela P, et al. Documento de consenso de la Sociedad Española de Medicina Intensiva, Crítica, y Unidades Coronarias (SEMICYUC), la Sociedad Española de Otorrinolaringología y Cirugía de Cabeza y Cuello (SEORL-CCC) y la Sociedad Española de Anestesiología y Reanimación (SEDAR) sobre la traqueotomía en pacientes con COVID-19. Acta Otorrinolaringol Esp. 2020: S0001.

38. Considerations of tracheostomy. Irish Head and Neck Society. Abril 17, 2020. 